\title{
An In Vitro Evaluation of 3D-printed Provisional Restoration Marginal Adaptation on Diverse Finish Lines
}

\author{
Roushan Kumar ${ }^{1}$, Mahesh Suganna², Rafi Ahmed ${ }^{3}$, Maneesh K Rajan ${ }^{4}$
}

\begin{abstract}
Aim and objective: Interim restorations are a crucial part of fixed prosthodontic treatment. Frequently such prostheses are used to serve the purpose of the therapeutical strength of a specific treatment plan or the form and function of the designed definitive prosthesis. The present study has been projected to value the marginal fit of two dissimilar interim restorations fabricated by 3D printing and milling technique on two different finish lines (shoulder and chamfer).

Materials and methods: Two typodont models of maxillary central incisors comprised prepared for the full coverage of dental restorations. Each typodont model was prepared using two different finish lines one with chamfer (C) and the other shoulder (S). Both the preparations were digitally scanned and exported as Standard Tessellation Language (STL) to software for the designing and fabrication of the full coverage dental restorations. Total 24 restorations were fabricated, 12 were 3D printed and 12 were computer-aided design/computer-aided manufacture (CAD/CAM) milled. In both types of restorations, half was fabricated on the chamfer finish line and the remaining half was fabricated on the shoulder finish line. Descriptive and independent $t$-test was done for intergroup comparison. Quantitative data were summarized using mean and standard deviation with a significance level of $p<0.001$.

Results: In this study, it was found that on shoulder finish line CAD/CAM-milled restoration showed the maximum mean marginal gap in the cervical margin as $77.42 \mu \mathrm{m}$, while 3D printed showed a gap of $41.08 \mu \mathrm{m}$. On the shoulder finish line 3D-printed restoration showed a maximum mean marginal gap in the cervical margin as $48.25 \mu \mathrm{m}$, while CAD/CAM milled showed a gap of $89.00 \mu \mathrm{m}$. There was a statistically highly significant difference present in the marginal gap between CAD/CAM and 3D printing at various locations in restoration with shoulder/ chamfer finish line. There was no statistically significant difference in the marginal gap in both finish lines irrespective of the fabrication method. Conclusion: Both the shoulder and chamfer finish lines were equally effective. Restorations fabricated by 3D printing had less marginal gap than CAD/CAM milling but both values were within the clinically acceptable limit.

Keywords: 3D printing, Chamfer, Computer-aided design/computer-aided manufacture milling, Marginal Discrepancy, Shoulder. International Journal of Prosthodontics and Restorative Dentistry (2021): 10.5005/jp-journals-10019-1313
\end{abstract}

\section{INTRODUCTION}

The accurate fit of the restoration is the major determinant for its longevity and success. Accuracy in each step of fabrication of the restoration is the primary requisite for an accurate final restoration. Thus, accuracy in impression, interocclusion record, interim restoration, and final prosthesis fabrication process is highly desirable. Focus has always been on the accuracy of impression and the final restoration. ${ }^{1}$

For years, there has been gradual upgradation in each step of the prosthodontic procedure. The influence of digitalization is seen from impression-making techniques to final prosthesis fabrication. But the procedure of interim restoration fabrication is still based on conventional technique and has not changed much over the years. Often such prostheses are used to assist in the determination of the therapeutic effectiveness of a specific treatment plan or the form and function of the planned definitive prosthesis. The requirements of an interim restoration are essentially the same as for the definitive restoration, apart from longevity and possibly the sophistication of color. ${ }^{2}$

The term 3D printing is generally used to describe a manufacturing approach that builds objects one layer at a time, adding multiple layers to form an object. This process is more correctly described as additive manufacturing and is also referred to as rapid prototyping. ${ }^{3}$

The introduction of computer-aided design/computer-aided manufacture (CAD/CAM) technology in dentistry has allowed the
1,3,4 Department of Prosthodontics and Crown and Bridge, Mithila Minority Dental College and Hospital, Darbhanga, Bihar, India

${ }^{2}$ Department of Prosthodontics and Dental Technology, Riyadh Elm University, Riyadh, Kingdom of Saudi Arabia

Corresponding Author: Mahesh Suganna, Department of Prosthodontics and Dental Technology, Riyadh Elm University, Riyadh, Kingdom of Saudi Arabia, Phone: +91 9482517333, e-mail: maheshgolgeri@gmail.com

How to cite this article: Kumar R, Suganna M, Ahmed R, et al. An In Vitro Evaluation of 3D-printed Provisional Restoration Marginal Adaptation on Diverse Finish Lines. Int J Prosthodont Restor Dent 2021;11(2): 82-87.

Source of support: Nil

Conflict of interest: None

shaping of high-performance material that could not otherwise be easily shaped to form a dental restoration. ${ }^{4}$ Several researchers have criticized the marginal fit of these restorations. ${ }^{5,6}$ However, improvements in the CAD/CAM system and software have made the fit more acceptable through precise operating procedures. ${ }^{7}$ This CAD/CAM system can be explored for the fabrication of the interim restoration.

The success of dental restoration is determined by three main factors: esthetic value, resistance to fracture, and marginal adaptation. Inadequate fit leads to plaque accumulation, which 
increases the risk of carious lesions, and can cause microleakage and endodontic inflammation. Plaque accumulation may also cause periodontal diseases, especially with subgingival margins. Finally, misfit could contribute to cement dissolution. ${ }^{8,9}$

The factors that have been documented to influence the marginal adaptation of dental restoration are the preparation design, location, and design of finish line preparation, restorative material, fabrication method, impression material, and technique. The clinical goal of interim prosthesis restoration is to have a minimal marginal gap, protect the tooth, and prevent carries and poor gingival health.

The present study has been designed to evaluate the marginal fit of two different interim restorations fabricated by 3D printing and milling technique on two different finish lines (shoulder and chamfer).

\section{Materials and Methods}

This study was conducted in the Department of Prosthodontics and Crown and Bridge of Mithila Minority Dental College and Hospital, Darbhanga, Bihar.

\section{Study Design}

Two typodont models of maxillary central incisors were prepared for full coverage of dental restorations. Each typodont model was prepared using two different finish lines. One central incisor was prepared using chamfer (C) and the other central incisor was prepared using shoulder (S). Both the preparations were digitally scanned and then scanned data were exported as Standard Tessellation Language (STL) to CAD/CAM software for the designing and fabrication of the full coverage dental restorations using polymethyl methacrylate (PMMA). Total 24 restorations were fabricated; 12 were 3D printed and 12 were CAD/CAM milled. In both types of restorations, half were fabricated on the chamfer finish line and the remaining half were fabricated on the shoulder finish line. The categorization of fabricated restorations is as follows:

- Group SM: shoulder finish line fabricated by CAD/CAM milling $(n=6)$.

- Group SP: shoulder finish line fabricated by 3D printing $(n=6)$.

- Group CM: chamfer finish line fabricated by CAD/CAM milling $(n=6)$.

- Group CP: chamfer finish line fabricated by 3D printing $(n=6)$.

Restorations were seated on respective prepared typodont models without cementation. The accuracy of all the restoration was assessed by observing them under an optical microscope and measuring the marginal gap at the prefixed point with the help of compatible software. The accumulated data are subjected to statistical analysis.

\section{Tooth Preparation}

The typodont models were prepared following the basic principles of tooth preparation. All models were prepared with $2 \mathrm{~mm}$ incisal reduction. Two different finish line designs were used $1.2 \mathrm{~mm}$ chamfer (C) and shoulder (S). All teeth were prepared with the use of silicon index (Varioline addition silicon; Kulzer), together with the use of magnifying loupe (Zumax $\times 3.5)$ for better visualization during tooth preparation. The preparations were done using a diamond bur kit under continuous water flow. All the preparation protocols were followed during tooth preparation with $2 \mathrm{~mm}$ incisal reduction, $1 \mathrm{~mm}$ facial and lingual shoulder and chamfer cervical margin preparation, $1.5 \mathrm{~mm}$ facial and lingual axial wall reduction and lingual concavity above cingulum. The finishing of the prepared tooth was done by using the finishing diamond burs. Overall, a total of 12 models were prepared with the shoulder finish line and 12 with a chamfer finish line.

\section{Restoration Design and CAD/CAM Fabrication}

All the scanning of typodont and fabrication of restorations for 3D printing (Confident Dental Laboratory, Bengaluru) were done. Prepared models were scanned (Intelli-denta master easy dental 3D scan) with a desktop optical scanner. The scanner was calibrated following the manufacturer's instructions. A thin layer of anti-reflective powder coating was sprayed from a fixed distance before performing an optical scanning procedure (Easy scan spray; Alphadent). It helps in displaying the preparation boundaries. The individual dies were secured firmly in a multi-die holder (Fig. 1) and placed in the Intelli-denta 3D scanner. After minor adjustments in digital impression (Fig. 2), digital files were generated using Exocad version 2016 and exported in STL format to CAM machine with software DS Cam, for the designing of restorations.

Milled restorations were fabricated using 5 -axis dry milling machine Intelli-Denta (MB Maschinen, Germany). All milled restorations were fabricated using $A 2$ shade of PMMA disk (Ruthinium disc; Ruthinium Dental Product Pvt. Ltd). The tool path of the specimen design was calculated by computer-assisted manufacturing software (DSCAM). Two kinds of burs of diameter 1 and $2 \mathrm{~mm}$ (conifil zirconia; VPT) were used during the milling procedure. Then, the specimens were ground and polished with silicon carbide paper of grain size 600 and 1,200 grit on a rotary machine with water cooling. All the above process was carried as per the manufacturer's recommendations. All specimens were stored separately for further study.

\section{Fabrication of Restorations by 3D Printing}

A digital impression of prepared typodont was exported to a 3D printing machine by CAD software through STL technique. Printed restorations were fabricated by using liquid photopolymer resin (Formlabsinc, Somerville, MA, USA). The specimens of the $3 D$-printed restorations were manufactured using a digital light processing (DLP) 3D printer (CARA Print 4.0; Kulzer) (Fig. 3). The laser was controlled by the digital micromirror. Then, the entire layer of liquid photopolymer resin was polymerized at once. Printed restorations were washed with $100 \%$ isopropyl alcohol in the

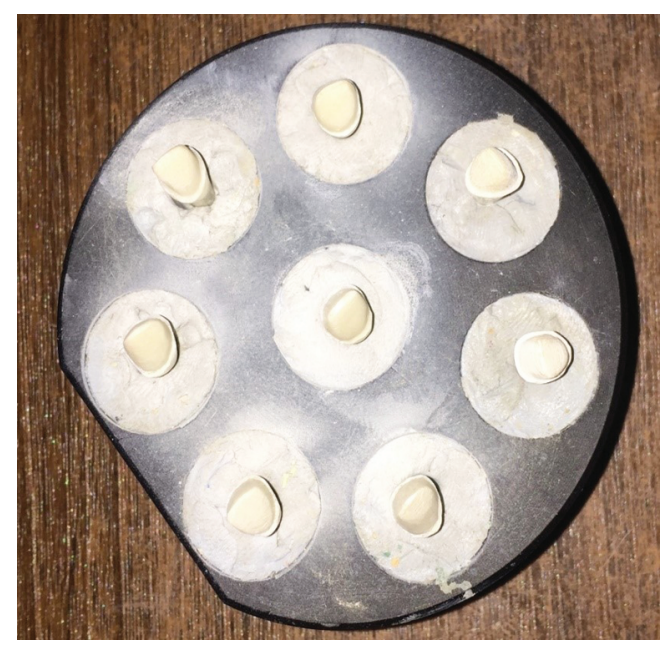

Fig. 1: Multi-die holder 


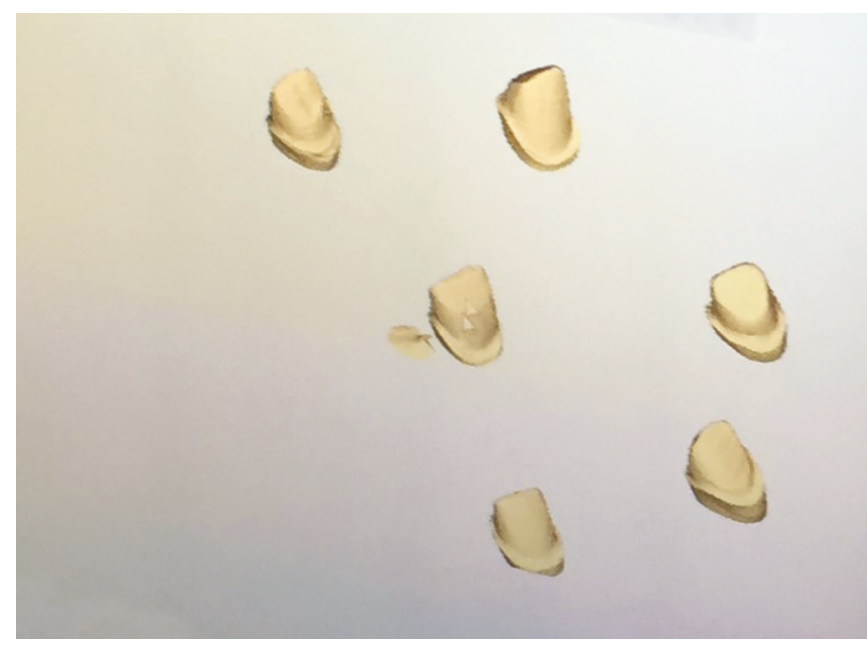

Fig. 2: Digital impressions

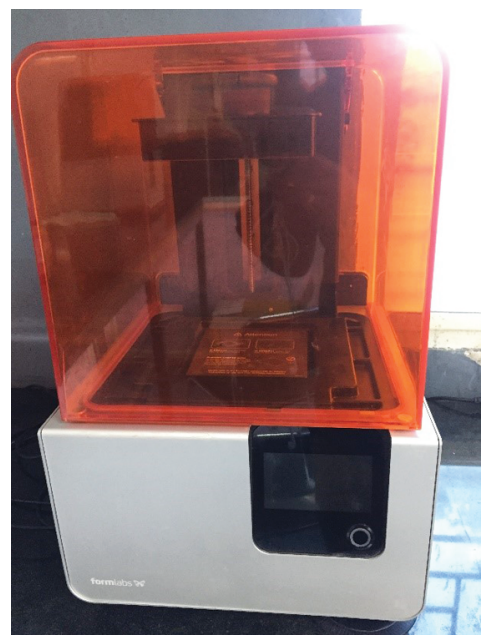

Fig. 4: Curing of restorations using curing unit

washing unit (Formlabs washing unit), to remove excessive resin monomers. In the final stage, the specimen underwent a curing process for 120 minutes using a curing unit (Form Cure; Formlabs) (Fig. 4). Then, the specimens were ground and polished with silicon carbide paper of grain size 600 and 1,200 grit on a rotary machine with water cooling. All specimens were stored separately for further study.

\section{Measurement of Marginal Gap}

All restorations were seated on respective prepared typodont without cementation. Marginal gaps were measured on each surface of restoration-facial, palatal, mesial, and distal. Arbitrarily two points are taken on each surface at the cervical margin. In this way, a total of eight measurements were taken for each restoration. The mean marginal gap on one surface was calculated by taking an average of the measured marginal gap on two different points of the respective surface. Typodont along with respective interim restoration was fixed on the stage of optical microscope (Zeiss; Axiocam 503 color) (Fig. 5), with the help of clay. The power of the aperture of this microscope was $10 \times$ and the lens used in measurement was $5 \times$. Total magnification of $50 \times$ was obtained while measuring the marginal gap under this optical microscope.

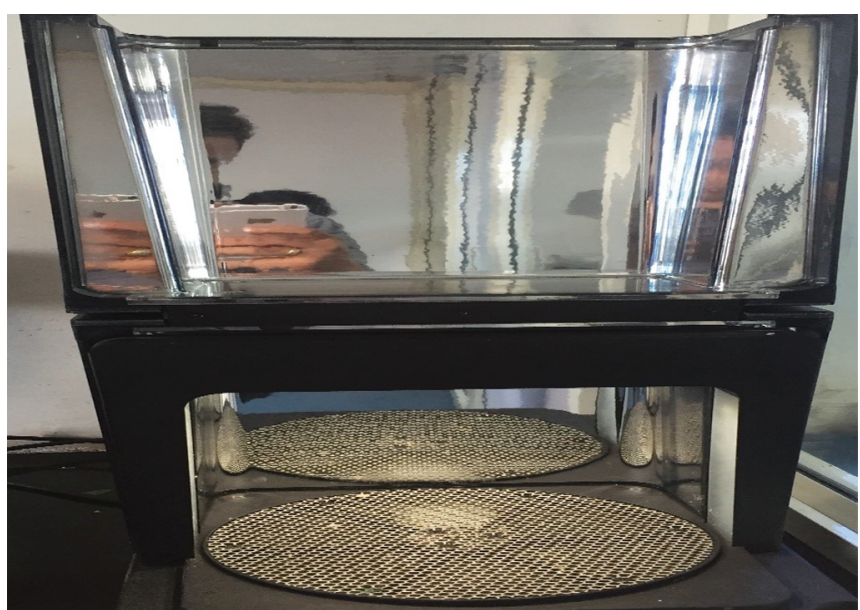

Fig. 3: Fabrication of 3-D printed restorations using 3D printing unit

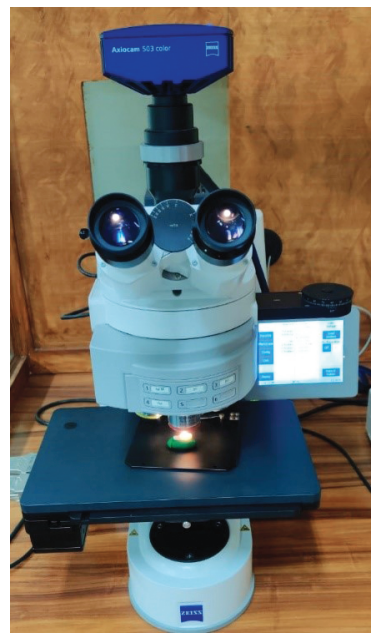

Fig. 5: Evaluation of marginal gap under optical microscope

An optical microscope was attached with a desktop having software axiocam axiovision 64-bit. Marginal gaps were visible as white or cream channels on the monitor of the desktop.

Descriptive statistics were performed in the present study and data obtained were subjected to the statistical package for the social sciences (SPSS) version 23. A descriptive, independent $t$-test was done for inter-group comparison. Quantitative data were summarized using mean and standard deviation. The statistical power of the study was $95 \%$.

\section{Results}

The compiled data were analyzed and the following observations in the form of tables and graphs were made. Table 1 and Figure 6 compared the marginal gaps in different surfaces of the restoration fabricated by using different techniques on teeth prepared with a shoulder finish line. In CAD/CAM technique, the maximum mean marginal gap was seen in the palatal cervical margin $(77.42 \mu \mathrm{m})$, while the lowest was seen in the facial margin $(70.25 \mu \mathrm{m})$. In 3D printing, the facial margin has the lowest marginal gap $(38.50 \mu \mathrm{m})$ while the distal surface was having a higher marginal gap (41.08 $\mu \mathrm{m})$. Overall, there was an increased marginal gap seen in interim restoration fabricated by using CAD/CAM. There was a statistically highly significant difference $(p<0.001)$ present in the marginal 
Table 1: Comparison of the marginal gap in the shoulder finish line

\begin{tabular}{|c|c|c|c|c|c|c|}
\hline \multirow[b]{2}{*}{ Group } & \multirow[b]{2}{*}{$N$} & \multicolumn{2}{|c|}{ CAD/CAM } & \multicolumn{2}{|c|}{$3 D$ printing } & \multirow[b]{2}{*}{$p$ value } \\
\hline & & Mean & $S D$ & Mean & $S D$ & \\
\hline Facial & 6 & 70.25 & 5.96 & 38.50 & 2.12 & $<0.001^{*}$ \\
\hline Mesial & 6 & 75.08 & 6.08 & 38.83 & 3.97 & $<0.001^{*}$ \\
\hline Palatal & 6 & 77.42 & 5.34 & 40.50 & 4.01 & $<0.001^{*}$ \\
\hline Distal & 6 & 76.58 & 6.92 & 41.08 & 3.57 & $<0.001^{*}$ \\
\hline
\end{tabular}

*Highly significant $(p<0.001)$

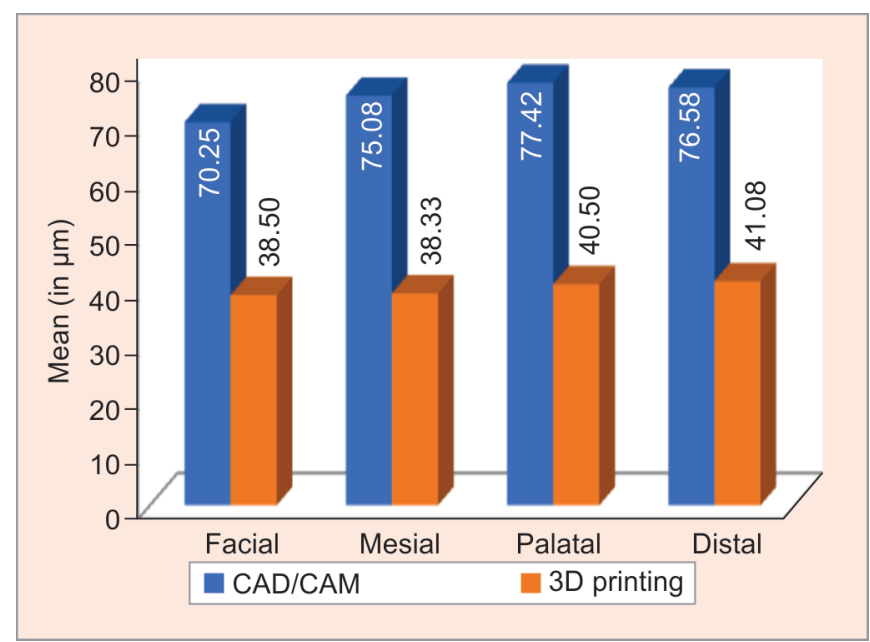

Fig. 6: Comparison of the marginal gap of interim restoration fabricated on the shoulder finish line

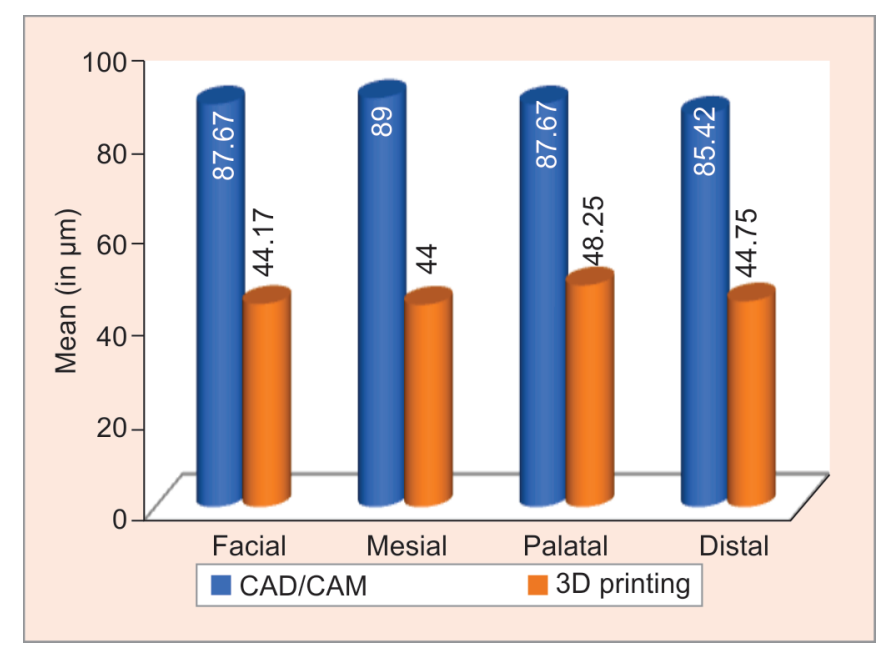

Fig. 7: Comparison of the marginal gap of interim restoration fabricated on the chamfer finish line

Table 2: Comparison of the marginal gap in the chamfer finish line

\begin{tabular}{|c|c|c|c|c|c|c|}
\hline \multirow[b]{2}{*}{ Group } & \multirow[b]{2}{*}{$N$} & \multicolumn{2}{|c|}{ CAD/CAM } & \multicolumn{2}{|c|}{$3 D$ printing } & \multirow[b]{2}{*}{$p$ value } \\
\hline & & Mean $(\mu m)$ & $S D$ & Mean $(\mu m)$ & $S D$ & \\
\hline Facial & 6 & 87.67 & 3.16 & 44.17 & 4.11 & $<0.001^{*}$ \\
\hline Mesial & 6 & 89.00 & 1.79 & 44.00 & 6.12 & $<0.001^{*}$ \\
\hline Palatal & 6 & 87.67 & 2.18 & 48.25 & 4.47 & $<0.001^{*}$ \\
\hline Distal & 6 & 85.42 & 2.60 & 44.75 & 4.60 & $<0.001^{*}$ \\
\hline
\end{tabular}

*Highly significant $(p<0.001)$

gap between CAD/CAM and 3D printing at various locations in restoration with a shoulder finish line. Table 2 and Figure 7 compared the marginal gaps in different surfaces of the restoration fabricated by using different techniques on teeth prepared with the chamfer finish line. In CAD/CAM technique, the maximum mean marginal gap was seen in the mesial cervical margin (89.00 $\mu \mathrm{m})$, while the lowest was in the distal margin $(85.42 \mu \mathrm{m})$. In 3D printing, the facial margin had the lowest marginal gap $(44.00 \mu \mathrm{m})$, while the distal surface showed the highest $(48.25 \mu \mathrm{m})$. There was a statistically significant difference in the marginal gap between CAD/CAM and 3D printing at various locations in restoration with chamfer finish line $(p<0.001)$. Overall, there was a less marginal gap in restorations fabricated by $3 \mathrm{D}$ printing.

Comparison of marginal gap (Table 3 and Fig. 8) of interim restorations fabricated on shoulder and chamfer finish line irrespective of the fabrication technique. There was no statistically significant difference $(p>0.05)$ in the marginal gap in both finish lines. So, both the finish lines were equally effective. Comparison of overall mean marginal gap (Table 4 and Fig. 9) of the restorations. Mean marginal gap in restoration fabricated by CAD/CAM milling on the shoulder $(73.61 \mu \mathrm{m})$ and chamfer $(83.12 \mu \mathrm{m})$. While in restoration fabricated by 3D printing on the shoulder $(43.44 \mu \mathrm{m})$ and chamfer $(49.21 \mu \mathrm{m})$ marginal gap. A statistically highly significant $(p<0.001)$ difference was present in the marginal gap between CAD/CAM and $3 \mathrm{D}$ printing in both finish lines.

\section{Discussion}

Interim restorations are an essential part of fixed prosthodontic treatment. Interim restoration must be fabricated and applied over the prepared tooth until final or definitive prosthesis placement. Interim restorations are a critical component of fixed prosthodontic treatment, biologically and biomechanically. Interim restoration serves an important diagnostic role as a functional and esthetic try-in and as a blueprint for the design of the definitive prosthesis. Marginal fit of full-coverage crowns is a major requirement for the long-term success of restorations. A perfect marginal seal is one 
An In Vitro Evaluation of 3D-printed Provisional Restoration Marginal Adaptation on Diverse Finish Lines

Table 3: Intercomparison of the marginal gap on different surfaces of restoration fabricated on teeth with shoulder and chamfer finish line

\begin{tabular}{|c|c|c|c|c|c|c|}
\hline \multirow[b]{2}{*}{ Group } & \multirow[b]{2}{*}{$N$} & \multicolumn{2}{|c|}{ Shoulder } & \multicolumn{2}{|c|}{ Chamfer } & \multirow[b]{2}{*}{$p$ value } \\
\hline & & Mean $(\mu m)$ & $S D$ & Mean $(\mu m)$ & $S D$ & \\
\hline Facial & 12 & 54.375 & 17.1214 & 65.917 & 22.9840 & $0.178 \mathrm{NS}$ \\
\hline Mesial & 12 & 56.958 & 19.5535 & 66.500 & 23.8909 & $0.296 \mathrm{NS}$ \\
\hline Palatal & 12 & 58.958 & 19.7984 & 67.958 & 20.8561 & $0.290 \mathrm{NS}$ \\
\hline Distal & 12 & 58.833 & 19.2677 & 65.083 & 21.5342 & 0.462 NS \\
\hline Overall & 12 & 58.520 & 17.1700 & 66.160 & 20.0900 & $0.328 \mathrm{NS}$ \\
\hline
\end{tabular}

NS, Not significant $(p>0.05)$

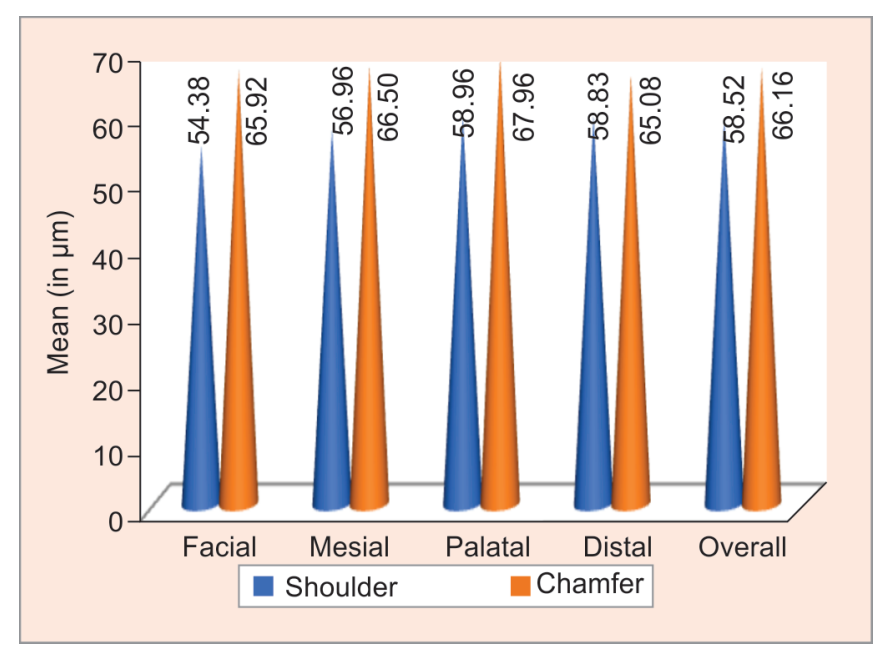

Fig. 8: Intercomparison of the marginal gap on different surfaces of restoration fabricated on teeth with shoulder and chamfer finish line

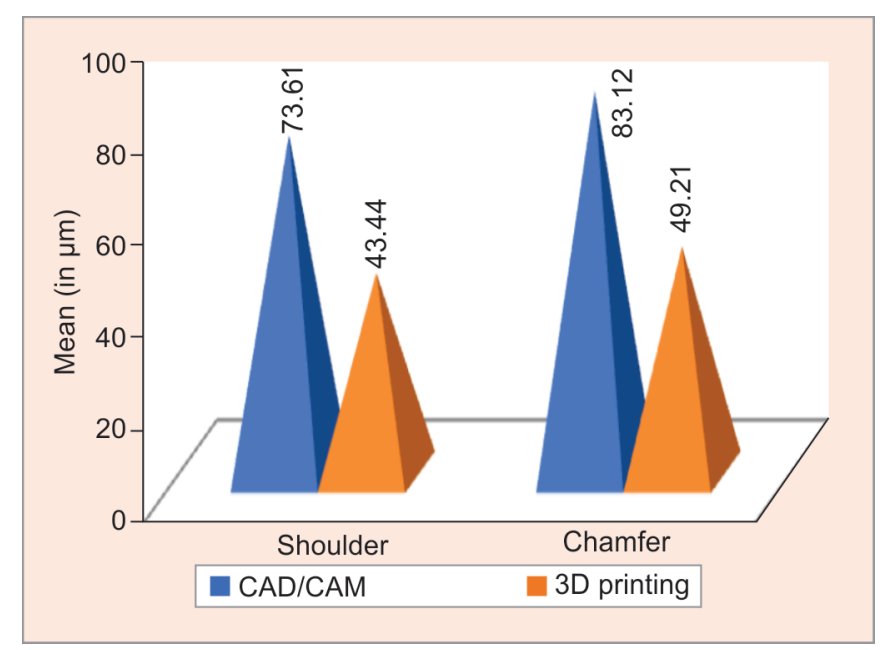

Fig. 9: Comparison of the mean marginal gap on shoulder and chamfer finish line fabricated by CAD/CAM and 3D printing

Table 4: Comparison of overall mean marginal gap

\begin{tabular}{|c|c|c|c|c|c|c|}
\hline \multirow[b]{2}{*}{ Group } & \multirow[b]{2}{*}{$N$} & \multicolumn{2}{|c|}{$C A D / C A M$} & \multicolumn{2}{|c|}{$3 D$ printing } & \multirow[b]{2}{*}{$p$ value } \\
\hline & & Mean $(\mu m)$ & $S D$ & Mean $(\mu m)$ & $S D$ & \\
\hline Shoulder & 6 & 73.61 & 5.00 & 43.44 & 8.83 & $<0.001^{*}$ \\
\hline Chamfer & 6 & 83.12 & 10.58 & 49.21 & 9.32 & $<0.001^{*}$ \\
\hline
\end{tabular}

*Highly significant $(p<0.001)$

of the most important technical factors for the long-term success of any restoration. ${ }^{2}$

A considerable marginal opening concedes more plaque accumulation, gingival sulcular fluid flow, and bone loss, recurrent caries, periodontal disease, and a decrease in the longevity of the prosthetics restorations. This kind of problem may be complicated by fixed partial restorations with vital abutments. ${ }^{10}$

A restoration, definitive or provisional, is termed successful when it exhibits good marginal and internal fit and is strong enough to withstand the oral environment. This is critical in the case of marginal adaptation of provisional restoration because the poor marginal fit can lead to inflammation of periodontal tissue, a situation that can hamper and postpone the fit of definitive restorations. Provisional restorations are used as an intermediate stage for short- or long-term placement on teeth between the time of tooth preparation until definitive indirect restorations are fabricated and placed. ${ }^{2}$

The purpose of this study was to evaluate and compare the marginal fit of 3D-printed and CAD/CAM milled interim restorations fabricated on two different finish lines, chamfer and shoulder. Based on the results, the null hypothesis that no difference exists in marginal fit between 3D printing and CAD/ CAM milled restorations on different finish lines was rejected. In the literature, several methods are reported to evaluate the marginal fit and adaptation of the different restorations on it. In this study, the optical microscope was used for measuring the marginal gap. This approach offers several advantages of being non-destructive and allows for quantitative measurements in three dimensions. However, one major shortcoming of this method is, all measurements were done without cementation or any pressure application on restoration apically, which of course is not a true representation of the actual clinical situation. Nonetheless, it has been shown that the use of cement may lead to improper seating of the restoration and thus increases the marginal and internal gap.

In this study, the results showed that 3D-printed restorations exhibit significantly lower marginal and internal gap values rather than CAD/CAM milled counterparts. The inferior marginal fit of milled restorations may be attributed to errors resulting from the tolerance of milling burs. 
In this study, while the shoulder finish line was used, it was found that there is almost the same marginal discrepancy present on each surface if the restoration fabrication technique was the same. But, the maximum marginal gap in CAD/CAM milled restoration was $77.42 \mu \mathrm{m}$ while in $3 \mathrm{D}$-printed restoration it was found $41.08 \mu \mathrm{m}$. There was a statistically highly significant difference present in the marginal gap between CAD/CAM and 3D printing at various points in restorations fabricated on the shoulder finish line. In the present study, marginal gaps in restoration fabricated on shoulder finish lines were within the clinically acceptable limit. But overall, there was a greater marginal gap in restorations fabricated by CAD/CAM milling.

In the present study in restorations fabricated on the chamfer, finish lines showed slightly increased marginal discrepancy than the shoulder finish line. The maximum marginal gap in CAD/CAM milled restorations was $89.00 \mu \mathrm{m}$ and in the 3D-printed restoration was $48.25 \mu \mathrm{m}$. There was a less marginal gap in restorations fabricated by $3 \mathrm{D}$ printing.

Statistical analysis showed that there was no significant difference in the marginal gap in both finish lines. So, both the finish lines were equally effective. Euán et al. ${ }^{11}$ also found in their study that there is no statistically significant difference in the marginal gap when the restorations were fabricated on different finish lines. Comlekoglu et al. ${ }^{12}$ also found in their study that both shoulder and chamfer finish lines exhibit the same type of marginal gap between restoration and prepared tooth. Our result is in concurrence with the findings from the above authors. But in the study conducted by Cho et al., ${ }^{13}$ it was found that marginal gaps were greater in the chamfer finish line when compared to the shoulder finish line.

In this study, a statistically highly significant difference was present in the marginal gap between CAD/CAM and 3D printing in both finish lines. It means restorations fabricated by $3 D$ printing had a less marginal gap irrespective of the finish line designs. This is in accordance with the results of the study of Alharbi et al. ${ }^{14}$ concluded that less marginal discrepancies were present in restorations fabricated by 3D printing.

From the present study, it can be inferred that the restorations fabricated by CAD/CAM milling are having a larger marginal gap than 3D-printed restorations irrespective of finish line designs. Boitelle et al. ${ }^{15}$ did a meta-analysis and found that most authors agree that the marginal gap between prepared tooth and restoration below $120 \mu \mathrm{m}$ are clinically acceptable. In the present study, the maximum marginal gap on shoulder and chamfer finish lines is in a clinically acceptable limit. Hence, it can be concluded that clinically acceptable restorations can be obtained by CAD/ CAM milling or 3D printing technique on either of the finish line design.

\section{Conclusion}

Both the shoulder and chamfer finish lines were equally effective. Restorations fabricated by 3D printing had less marginal gap than CAD/CAM milling but both values were within the clinically acceptable limit.

\section{References}

1. Nawafleh NA. Accuracy and reliability of methods to measure marginal adaptation of crowns and FDPs: a literature review. J Prosthet dent 2013;22(5):419-428. DOI: 10.1111/jopr.12006.

2. Gratton DG, Aquilino SA. Interim restorations. Dent Clin N America 2004;48(2):487-497. DOI: 10.1016/j.cden.2003.12.007.

3. Dawood A, Marti BM, Sauret-Jackson V, et al. 3D printing in dentistry. Br Dent J 2015;219(11):521-529. DOI: 10.1038/sj.bdj.2015.914.

4. Jedynakiewicz NM, Martin N. CEREC: science, research, and clinical application. The Compend Contin Edu Dentis 2001;22(6 Suppl):7-13.

5. Hickel R, Dasch W, Janda R, et al. New direct restorative materials. FDI commission project. Int Dent J 1998;48(1):3-16. DOI: 10.1111/j.1875595x.1998.tb00688.x.

6. Bayne SC, Heymann HO. CAD/CAM in dentistry: present and future applications. Quintess Int (Current Concepts) 1996;27:431-433.

7. Tsitrou EA, Northeast SE, van Noort R. Evaluation of the marginal fit of three margin designs of resin composite crowns using CAD/CAM. J Dentis 2007;35(1):68-73. DOI: 10.1016/j.jdent.2006.04.008.

8. Borba M, Cesar PF, Griggs JA, et al. Adaptation of all-ceramic fixed partial dentures. Dent Mater 2011;27(11):119-126. DOI: 10.1016/j. dental.2011.08.004.

9. Contrepois $M$, Soenen A, Bartala M, et al. Marginal adaptation of ceramic crowns: a systematic review. J Prosthet Dent. 2013. ; 110(6):447-454.e10. DOI: 10.1016/j.prosdent.2013.08.003.

10. Liang S, Yuan F, Luo X, et al. Digital evaluation of absolute marginal discrepancy: a comparison of ceramic crowns fabricated with conventional and digital techniques. J Prosthet Dent 2018;120(4):525529. DOI: 10.1016/j.prosdent.2017.10.014.

11. Euán R, Figueras-Álvarez O, Cabratosa-Termes J, et al. Comparison of the marginal adaptation of zirconium dioxide crowns in preparations with two different finish lines. J Prosthet Dent 2012;21(4):291-295. DOI: 10.1111/j.1532-849X.2011.00831.x.

12. Comlekoglu M, Dundar M, Ozcan M, et al. Influence of cervical finish line type on the marginal adaptation of zirconia ceramic crowns. Operat Dentis 2009;34(5):586-592. DOI: 10.2341/08-076-L.

13. Cho L, Choi JM, Yi YJ, et al. Effect of finish line variants on marginal accuracy and fracture strength of ceramic optimized polymer/fiberreinforced composite crowns. J Prosthet Dent 2004;91(6):554-560. DOI: 10.1016/j.prosdent.2004.03.004.

14. Alharbi N, Alharbi S, Cuijpers VM, et al. Three-dimensional evaluation of marginal and internal fit of 3D-printed interim restorations fabricated on different finish line designs. J Prosthod Res 2018;62(2):218-226. DOI: 10.1016/j.jpor.2017.09.002.

15. Boitelle $P$, Mawussi $B$, Tapie $L$, et al. A systematic review of CAD/CAM fit restoration evaluations. J Oral Rehabilit 2014;41(11):853-874. DOI: 10.1111/joor.12205. 